various nuclear disaster, new concept and new comprehensive disaster medical system is necessary as well as effective utilization of pre-existing resources.

Prehosp Disaster Med 2017;32(Suppl. 1):s3-s4 doi:10.1017/S1049023X17000371

The New Radiation Emergency Medical System in Japan: Lessons from the Fukushima Nuclear Plant Accident Hitoshi Yamamura, Shinya Yaguchi, Katsubiro Itoh Dept Of Disaster And Critical Care Medicine, Hirosaki University, Graduate School of Medicine, Hirosaki Aomori/Japan

Study/Objective: Our aim was to clarify the new radiation emergency medical system in Japan, and the related activities at our hospital after the Fukushima No. 1 Nuclear Power Plant accident. Background: The radiation accident at Fukushima No. 1 Nuclear Power Plant occurred on March 11, 2011. After this accident, the Japanese radiation medical system was in a state of confusion because health care workers had no knowledge about radiation emergencies and there was no appropriate organization to handle the control of a radiation disaster.

Methods: The Japanese government created two special radiation medical centers after the accident. First was a Radiation Disaster Medical Care and general Support Center comprised of four hospitals, with the role of coordinating the radiation emergency medical assistant teams, treatment of radiation exposure patients, and training of the hospital staff in Radiation Emergency Medicine (REM). Second was an Advanced Radiation Emergency Medical Support Center comprised of five hospitals with an advisory role in dispensing advice about professional REM dissymmetry for internal exposure, special training for professional research, and knowledge about REM. Our hospital was designated as a member of the above two centers, and we investigated our related activities.

Results: Since our designation, we have rebuilt the REM system in our hospital. Our achievements mainly include education, the development of training contents for activities in our hospital, and lectures on REM for the hospital staff including the doctors, nurses, radiologists, laboratory technicians, and office employees. Hands-on training and lectures were given on REM for medical students. We have also participated in REM training on the national and prefatory levels.

Conclusion: It is important for us to educate all of the health care workers in our hospital about radiation emergencies, and to train professional staff who are familiar with both general disaster medical care and radiation emergency medical treatment. Prehosp Disaster Med 2017;32(Suppl. 1):s4

doi:10.1017/S1049023X17000383

Development and Effect of Personal Protective Equipment, Train-the-Trainer Program for Hospital Nurses Jiyoung Noh ${ }^{1}$, Hyun Soo Chung ${ }^{2}$, Minhong Choa ${ }^{1}$, Seunghrwan Kim ${ }^{1}$, Inkeun Kim ${ }^{1}$

1. Center For Disaster Relief, Training, And Research, Yonsei University Severance Hospital, Seoul/Republic of Korea

2. Emergency Medicine, Yonsei University College of Medicine, Seoul/Republic of Korea
Study/Objective: To develop a standardized Personal Protective Equipment (PPE), Train-the-Trainer Program for hospital nurses and to investigate the effect of the program.

Background: Despite the importance and perception of nurses in preparation for contaminated and/or infectious crisis, a standardized program to develop competencies is still lacking in Korea. Many hospitals train for protective equipment through large group lectures. Some institutions conduct handson training, but the educational contents and assessment tools are not standardized. PPE training is needed for all hospital personnel that has the potential to be in contact with patients. The number of hospital personnel mounts to more than 1,000, and it is very difficult to train everyone in a single place by few trained instructors. Therefore, it is important to train trainers to be competent in training PPE.

Methods: Staff from the Office of Infection Control, Office of Quality Improvement, Department of Emergency Medicine, Department of Nursing, and Center for Disaster Training gathered to develop a standardized training content and assessment tool. The tools were validated through the content validity index. After pilot testing, 44 nurses from five different departments were selected to become trainers. The educational intervention consisted of a 2-hour workshop. A pre- and postsurvey was conducted to evaluate the differences in perception and performances in personal protection (paired t tests). The statistical level of significance was set at 0.05 .

Results: Pre- and post-survey differences in perception for PPE knowledge and confidence were 5.3 to 8.4 and 5.3 to 8.3, respectively. Average performance points out of 10 was 9.1, and the observed points in the role of trainers was 9.0 out of 10 . All 44 participants passed the minimum passing score of 90 percentage.

Conclusion: A standardized train-the-trainer program for PPE was successfully developed, and the newly trained trainers will be performing their roles as trainers for PPE.

Prehosp Disaster Med 2017;32(Suppl. 1):s4

doi:10.1017/S1049023X17000395

\section{Middle East Respiratory Syndrome Coronavirus (MERS-CoV) Outbreak and National and Hospital}

\section{Response in Korea}

Soon-Joo Wang

Hallym University, Hwaseong/Republic of Korea

Study/Objective: The study objective is to understand the MERS-CoV outbreak outside the Middle East.

Background: The outbreak of Middle East Respiratory Syndrome Coronavirus (MERS-CoV) infection in the Republic of Korea started from the index case who developed fever after returning from the Middle East. He infected 26 cases in Hospital P, and consecutive nosocomial transmission proceeded throughout the nation. The author provided an epidemiologic description, the hospital response, and the first case of mortality from the outbreak.

Methods: Epidemiological research was performed by direct interview of the health care professionals, and reviewing medical records in the hospital where the first mortality occurs in Korea. 